\title{
Abnormal alterations of miR-1 and miR-214 are associated with clinicopathological features and prognosis of patients with PDAC
}

\author{
QING CHENG, LI-HUA HAN, HAI-JUAN ZHAO, HUI LI and JIAN-BING LI \\ Gerontology Department, Changzhi City People's Hospital, Changzhi, Shanxi 046000, P.R. China
}

Received January 14, 2016; Accepted July 27, 2017

DOI: $10.3892 / \mathrm{ol} .2017 .6819$

\begin{abstract}
Pancreatic ductal adenocarcinoma (PDAC) is a devastating malignant disease with a poor prognosis. PDAC is known to be difficult to diagnose at an early stage and to exhibit poor recurrence-free prognosis, but there is also a lack of effective treatment and limited knowledge of its biological characteristics. Therefore, there is an urgent requirement for an improved understanding of the cellular or molecular properties associated with PDAC, and to explore novel avenues for the diagnosis and treatment of this disease. In the present study, the microRNA (miRNA/miR) profiles of sera and tumor samples from patients with PDAC and healthy controls were investigated by miRNA microarray, and the potential role of miR-1 expression in PDAC was determined. A total of 43 patients attending the clinic diagnosed with PDAC at Changzhi City People's Hospital were invited to participate. Blood and surgical tumor samples were obtained for analysis by miRNA microarray and the reverse transcription-quantitative polymerase chain reaction (RT-qPCR). The surgical tumor tissue was additionally used to determine miRNAs status by in situ hybridization (ISH). The results of microarray revealed that: i) 27 miRNAs in the sera and 23 miRNAs in the tumor tissues obtained from patients with PDAC were different compared with their matched controls; ii) miR-1, miR-10b and miR-214 were significantly altered in the PDAC group, either in the sera or tumor tissue samples. Results from the RT-qPCR, which detected the levels of miRNAs in patients with PDAC, confirmed those obtained from the miRNA microarray. In particular, the results of the present study revealed that decreased miR-1 and increased miR-214 in the PDAC tissues were associated with the clinicopathological features and survival rates of patients with PDAC. The results of the present study indicated that miRNAs serve an important
\end{abstract}

Correspondence to: Dr Qing Cheng, Gerontology Department, Changzhi City People's Hospital, 105 North Street, Changzhi, Shanxi 046000, P.R. China

E-mail: chengqing1392015@126.com

Key words: pancreatic ductal adenocarcinoma, microRNA-1, microRNA-10b, microRNA-214, clinicopathological features, survival rate role in PDAC carcinogenic progression and supplied useful markers, including miR-1, miR-214 and miR-10b, for determining PDAC prognosis using noninvasive methods.

\section{Introduction}

Pancreatic cancer (PCC) is a highly malignant tumor that has a high rate of metastasis $(1,2)$. In the United States of America, PCC is one of the major causes of human mortality (1). In the USA, the total number of mortalities due to PCC are projected to increase markedly, and become the second leading cause of cancer-associated mortalities before 2030 (2). Among various types of PCC, pancreatic ductal adenocarcinoma (PDAC) accounts for $>90 \%$ of all pancreatic tumors. PDAC is a devastating and malignant disease with a poor prognosis, which is demonstrated through its 1-year survival rate of $\sim 18 \%$ for all stages of the disease $(3,4)$. In 2012 alone, PDAC resulted in 33,000 mortalities globally. Even in regions of the world with the best healthcare, only a minority of patients receive timely and prompt treatment, with a 5-year survival rate of $<22 \%$, and incidence is expected to rise with an aging population (5). PDAC is known for being difficult to diagnose at an early stage and it exhibits poor recurrence-free prognosis, but there is also a lack of effective treatment and limited knowledge of its biological characteristics (6,7). Therefore, there is an urgent requirement for improved understanding of the cellular or molecular properties associated with PDAC, and to explore novel avenues of diagnosis and treatment of this disease $(8,9)$.

MicroRNAs (miRNAs/miR) are involved in the inhibition of the translation and promotion of the degradation of their targeted mRNAs through binding to the 3' untranslated region (UTR). It also been demonstrated that miRNAs may act either as an oncogenic factor or suppressor during the development and progression of cancer tissues (10). Previous studies have suggested that miRNAs serve essential roles in tumor cell biological processes, including cell proliferation, differentiation, migration and invasion $(11,12)$. Previously, studies have also demonstrated that certain miRNAs are highly expressed in tumor tissues, whereas others are downregulated in tumor tissues $(13,14)$. These results suggest that alterations in the miRNA expression profiles of cancer tissues in comparison with normal tissues may be used in PCC diagnostics. There is an urgent requirement for novel and reliable biomarkers for the diagnosis and prognosis of PDAC. Circulating miRNAs have been extensively profiled in PDAC blood samples, but only a 
limited number of studies have performed adequate validation of candidate markers (15). In particular, the expression levels of miRNAs in the sera of patients with PDAC are unclear, and whether their alterations are associated with the severity and clinical outcome of PDAC are unknown.

The present study investigated the association between the expression status of miRNAs and carcinogenic progression in patients with PDAC. The miRNA profiles of sera and tumor tissues from patients with and without PDAC were explored using miRNA microarray analysis, and the possible roles of abnormal expression of miRNAs in PDAC were determined. A total of 43 patients attending the clinic in the Changzhi City People's Hospital (Changzhi, China) were invited to participate. Individual patients with PDAC were diagnosed using pathological examination by two independent pathologists. Among them, 7 patients were enrolled in the miRNA microarray analysis. To confirm the results of the miRNA microarray, the blood and surgical tumor samples of 43 patients were also obtained for reverse transcription-quantitative polymerase chain reaction analysis. The status of abnormal miRNAs expression in the tumor samples were also determined using in situ hybridization. The association between the expression status of the miRNAs and the clinicopathological features of PDAC was also analyzed.

\section{Materials and methods}

Study ethics. The present study complied with the International Ethical Declaration and was approved by the Human Ethics Committee and the Research Ethics Committee of Shanxi Province of China. Through the surgical consent form, patients were informed that the resected specimens were retained by the Changzhi City People's Hospital and may be used for scientific study, and that their privacy would be maintained.

Patients. The present study enrolled 43 patients (22 males and 23 females) attending the clinic in the Changzhi City People's Hospital, between February 2009 and January 2011. The patients ranged in age from 39 to 76 years, with a median age of 55 years. The median follow-up time was 60.2 months (mean \pm standard deviation, 60.2 \pm 2.36 months). No patients had received surgery or chemotherapy prior to the present study. The specimens were histopathologically verified as PDAC by two senior independent pathologists. Subsequently, the tumor samples and matched normal adjacent tissues, which were removed at least $0.5 \mathrm{~cm}$ distal to tumor margins, were obtained (16).

The biopsy samples were divided into two sections immediately following surgery. One fragment was immediately stored at $-80^{\circ} \mathrm{C}$ until nucleic acid isolation. In total, 7 randomly selected samples, and the matched sera samples, were prepared for miRNA microarray analysis. The remaining 36 samples were prepared for miRNA detection. The second remaining section of the 43 patients was fixed in $4 \%$ formaldehyde for 2 days and paraffin-embedded for in situ hybridization (ISH) examination. Formalin-fixed, paraffin-embedded tissues were sectioned $(5 \mu \mathrm{m})$ as described previously (17), and stained with hematoxylin and eosin (H\&E) per the manufacturer's protocol (Hematoxylin \& Eosin Staining kit, cat. no. C0105; Beyotime Institute of Biotechnology, Haimen, China). PDAC were classified according to histopathologic criteria as described previously (18).

In addition, 51 age- and sex-matched individuals from a large pool of individuals seeking a routine health checkup at the Changzhi City People's Hospital and exhibited no evidence of disease were recruited and served as the healthy control. They were processed for sera sample collection and the subsequent miRNA detection. Among them, 7 individuals were randomly selected as the normal controls for sera analysis using miRNA microarray.

RNA preparation and miRNA microarray. Following careful rinsing in ice-cold PBS, the sera or tissues were homogenized on ice in TRIzol ${ }^{\circledR}$ (Invitrogen; Thermo Fisher Scientific, Inc., Waltham, MA, USA). Total RNA was isolated using TRIzol ${ }^{\circledR}$ and a miRNeasy mini kit (Qiagen GmbH, Hilden, Germany) according to the manufacturer's protocol, which efficiently recovered all RNA species, including miRNAs. RNA quality and quantity was measured using a Nanodrop spectrophotometer (ND-1000; Nanodrop Technologies; Thermo Fisher Scientific, Inc.) and RNA integrity was determined by gel electrophoresis as described previously (19).

To detect miRNAs, 100 ng RNA was labeled and hybridized using the Human microRNA Microarray kit (release 12.0) (Agilent Technologies, Inc., Santa Clara, CA, USA) according to the manufacturer's protocol. Agilent microRNA microarrays (version 1.0) were employed for the analysis. Hybridization signals were detected using an Agilent DNA microarray scanner (G2505B; Agilent Technologies, Inc.) and the scanned images were analyzed using Agilent Feature Extraction software (version 10.10.1.1; Agilent Technologies, Inc.). All data were deposited in the National Center for Biotechnology Information Gene Expression Omnibus (GEO) and are accessible through GEO Series accession number GSE57555.

Sera sample preparation. The sera samples of patients with PDAC and the healthy controls were collected within $24 \mathrm{~h}$ following definite diagnosis and were processed immediately following collection. The sera were separated by centrifugation at $3,000 \times \mathrm{g}$ for $15 \mathrm{~min}$ at $4^{\circ} \mathrm{C}$, and then stored at $-80^{\circ} \mathrm{C}$ until analysis.

Reverse transcription-quantitative polymerase chain reaction $(R T$ - $q P C R)$. Total RNA from sera and tissue samples was prepared using TRIzol ${ }^{\circledR}$ and miRNeasy mini kit (Qiagen $\mathrm{GmbH}$ ) according to the manufacturer's protocol. cDNA was synthesized from total RNA using gene-specific primers from the TaqMan MicroRNA assay kit (Applied Biosystems; Thermo Fisher Scientific, Inc.), according to the manufacturer's protocol. qPCR of the miRNA was performed using an Applied Biosystems 7300 Sequence Detection system (Applied Biosystems; Thermo Fisher Scientific, Inc.). The $10 \mu \mathrm{l}$ PCR volume contained $0.67 \mu 1$ reverse transcription product, 1X TaqMan Universal PCR master mix, and $1 \mu \mathrm{l}$ of the primer and probe mixture, according to the TaqMan MicroRNA assay protocol. The threshold cycle data were determined using default threshold settings, according to the manufacturer's protocol. The threshold cycle was defined as the fractional cycle number at which the fluorescence exceeded the fixed threshold $(20,21)$. 
A

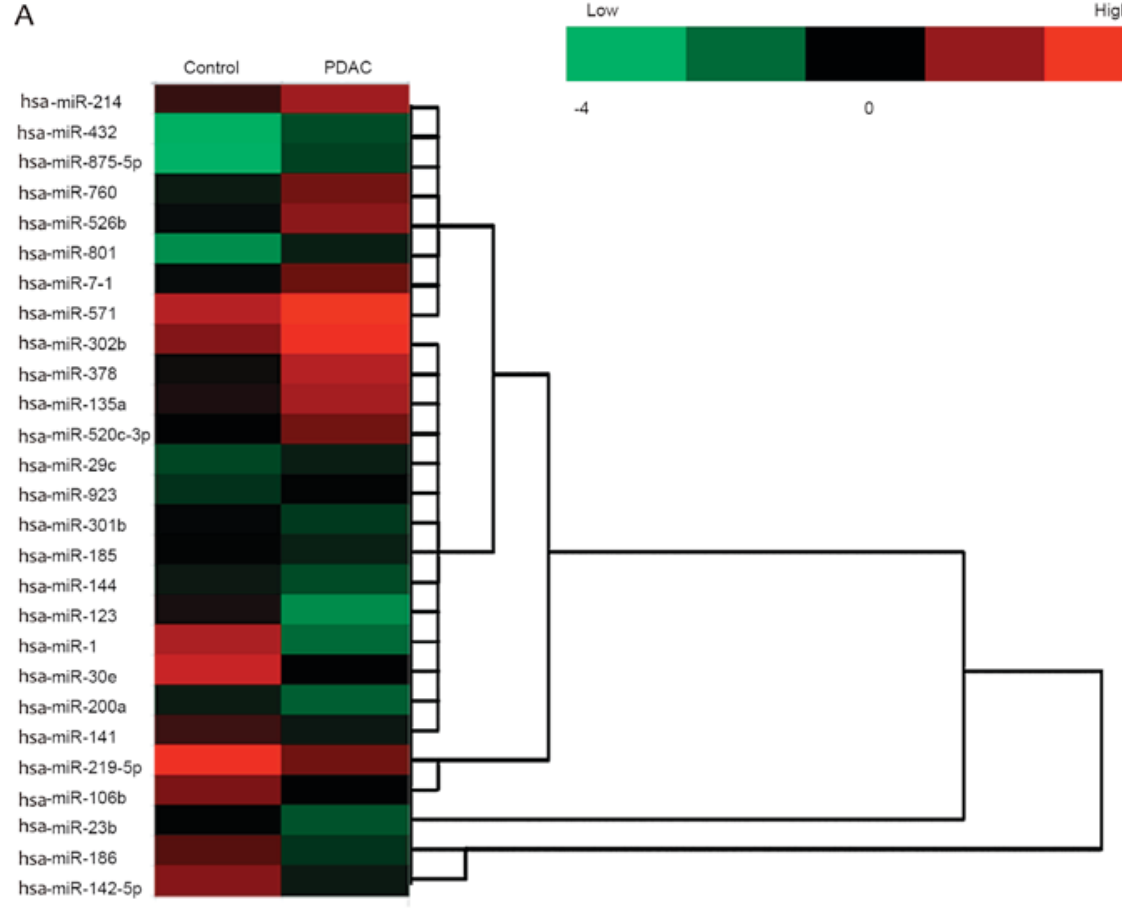

High

B

hsa-miR-10b

hsa-miR-575

hsa-miR-159a

hsa-miR-644

hsa-miR-581

hsa-miR-637

hsa-miR-935

hsa-miR-98

hsa-miR-513-3p

hsa-miR-5180

hsa-miR-382

hsa-miR-605

hsa-miR-19a

hsa-miR-1

hsa-miR-381

hsa-miR-93

hsa-miR-593-3p

hsa-miR-17

hsa-miR-101

hsa-miR-23a

hsa-miR-214

hsa-miR-653

hsa-miR-519d

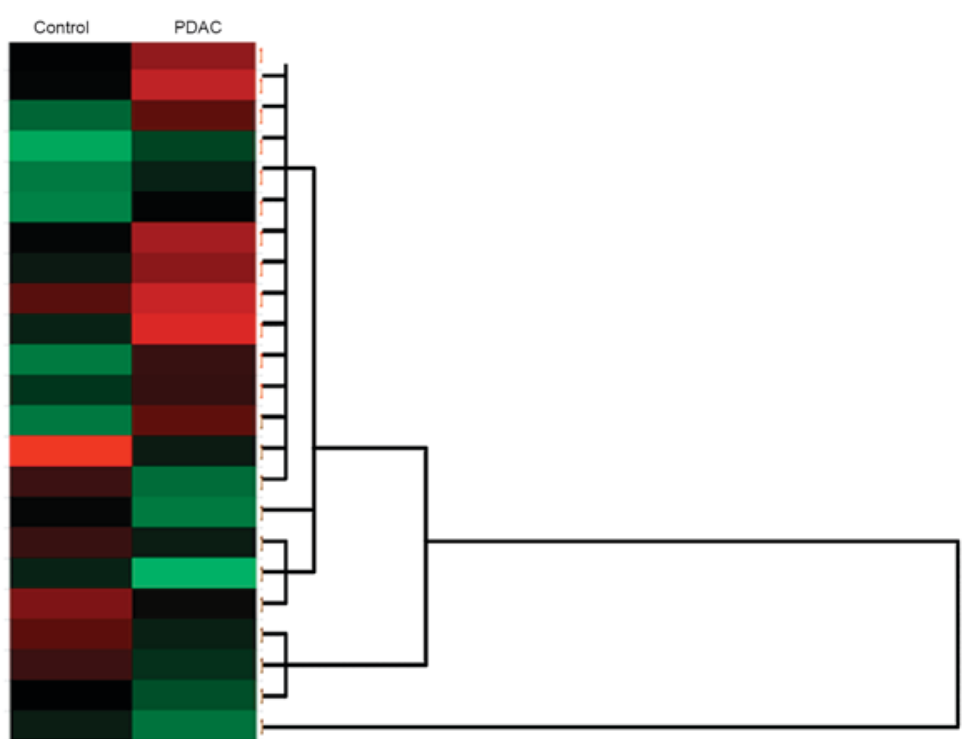

Figure 1. miRNA analysis of sera and tumor samples between PDAC and healthy controls. The heat-map identifiesthe significant differentially expressed miRNAs. miRNA expression in paired sera of patients with PDAC with healthy controls and tumor tissuesfrom patients with PDAC with the adjacent normal tissues were profiled. Red and green colors represent high and low expression, respectively. (A) Sera. (B) Tumor. miR, microRNA; PDAC, pancreatic ductal adenocarcinoma; hsa, Homo sapiens.

ISH. MicroRNA ISH Buffer and Controls kit (Exiqon A/S, Vedbaek, Denmark) were used for ISH in the present study, according to the manufacturer's protocol. The ISH detection of miR-1, miR-10b and miR-214 in tumor tissues were performed as described previously (22). Briefly, formalin-fixed paraffin-embedded PDAC tissues were cut into $4-\mu \mathrm{m}$ sections and deparaffinized (23). The probes for miR-1, miR-10b and miR-214 used in the present study were complementary to human mature miR-1, miR-10b and miR-214, respectively. For signal detection, anti-digoxigenin-alkaline phosphatase Fab fragments (1:800; Roche Applied Science, Penzburg, Germany; Roche Diagnostics Gmbh, Mannheim, Germany) were used as the primary antibody, and the slides were incubated with nitro blue tetrazolium/5-bromo-4-chloroindol-3-yl-phosphate solution (Roche Applied Science; Roche Diagnostics GmbH). Counterstaining was performed by Nuclear Fast Red (Chroma ATE Inc., Stuttgart, Germany).

Statistical analysis. Values are presented as the mean \pm standard error of the mean. Differences between groups were tested using a repeated-measure analysis of variance and post hoc test (least significant difference test or Dunnett's T3). Unpaired t-tests were used to compare baseline differences between groups. Associations between miRNAs expression (ISH staining) and other clinicopathological factors of the tumor were assessed using the two-sided Fisher's exact test for 
categorical variables, and a $\chi^{2}$ test was used to compare ordinal variables. The grading-associated data were analyzed using a Spearman's test. $\mathrm{P} \leq 0.05$ was considered to indicate a statistically significant difference, unless otherwise indicated. All analyses were performed using SPSS software (version 19.0; IBM Corp., Armonk, NY, USA).

\section{Results}

Different miRNA expression profiles in patients with PDAC. A fold change threshold of $\geq 2.0$ was used to identify differentially expressed miRNA between the two groups. In the sera from two groups, the expression levels of 27 miRNAs were different between patients with PDAC and healthy controls, in which 14 miRNAs were upregulated [Homo sapiens (hsa)-miR-214, hsa-miR-432, hsa-miR-875-5p, hsa-miR-760, hsa-miR-526b, hsa-miR-10b, hsa-miR-801, hsa-miR-571, hsa-miR-302b, hsa-miR-378, hsa-miR-135a, hsa-miR-520c-3p, hsa-miR-29c and hsa-miR-923], and 13 miRNAs were downregulated (hsa-miR-301b, hsa-miR-185, hsa-miR-144, hsa-miR-124, hsa-miR-30e, hsa-miR-200a, hsa-miR-141, hsa-miR-219-5p, hsa-miR-106b, hsa-miR-1, hsa-miR-23b, hsa-miR-186 and hsa-miR-142-5p; Fig. 1A). In tumor tissues obtained from the two groups, the expression levels of 23 miRNAs were different between PDAC tissues and adjacent normal controls, in which 12 miRNAs were upregulated (hsa-miR-10b, hsa-miR-575, hsa-miR-159a, hsa-miR-644, hsa-miR-581, hsa-miR-637, hsa-miR-935, hsa-miR-98, hsa-miR-513-3p, hsa-miR-518e, hsa-miR-382 and hsa-miR-214), and 11 miRNAs were downregulated (hsa-miR-1, hsa-miR-19a, hsa-miR-381, hsa-miR-93, hsa-miR-593-3p, hsa-miR-17, hsa-miR-101, hsa-miR-23a, hsa-miR-214, hsa-miR-653 and hsa-miR-519d; Fig. 1B). The present study focused on miR-1, miR-10b and miR-214, which were different in sera and in tumor samples between the PDAC and control groups (Fig. 2).

Quantification analysis of miRNAs. RT-qPCR methods were utilized to verify the microarray results. The expression of miR-1, miR-10b and miR-214 were detected in sera (from 43 patients with PDAC and 51 controls) and tumor tissues (from 43 patients with PDAC and the matched adjacent controls). The results demonstrated that, compared with that of healthy controls, miR-1 was decreased significantly in the sera from PDAC group (Fig. 3A; P $<0.05$ ). When compared with that of the matched normal adjacent tissues, the levels of miR-1 were also decreased in the tumor tissues from the PDAC group (Fig. 3A; $\mathrm{P}<0.05$ ). The quantitative analysis of miR-10b and miR-214 additionally confirmed that these miRs were upregulated in sera and tumor samples, respectively, between the PDAC and control tissues (Fig. 3B and C; $<<0.05$ ).

Abnormal expression of miR-1 associated with PDAC. miR-1, miR-10b and miR-214 RNA signals were detected in the tumor tissues of PDAC cells using ISH, and the cases that exhibited $>10 \%$ of the positive carcinoma cells were considered positive for miRNA ISH status in the present study (22). ISH detection demonstrated the positive staining of miR-1, miR-10b and miR-214 in normal adjacent (Fig. 4A-C) and PDAC tissues (Fig. 4D-F). The results demonstrated positive staining of
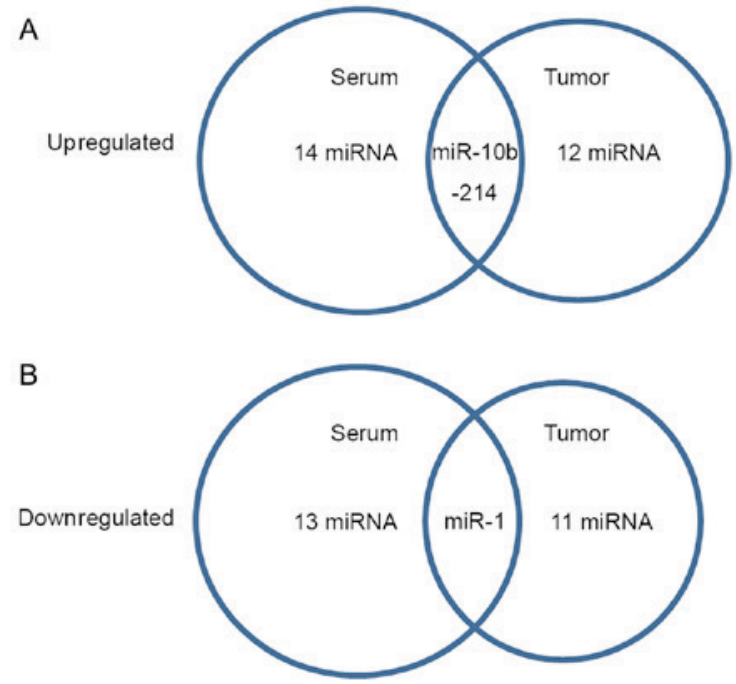

Figure 2. Differential miRNAs between PDAC and control samples. (A) Sera samples. (B) Tumor samples. The diagram demonstrates that among the differential miRNAs between the pancreatic ductal adenocarcinoma and control groups, miR-1, miR-10b and miR-214 were different either in the sera or in the tumor tissue samples. miR, microRNA; PDAC, pancreatic ductal adenocarcinoma.
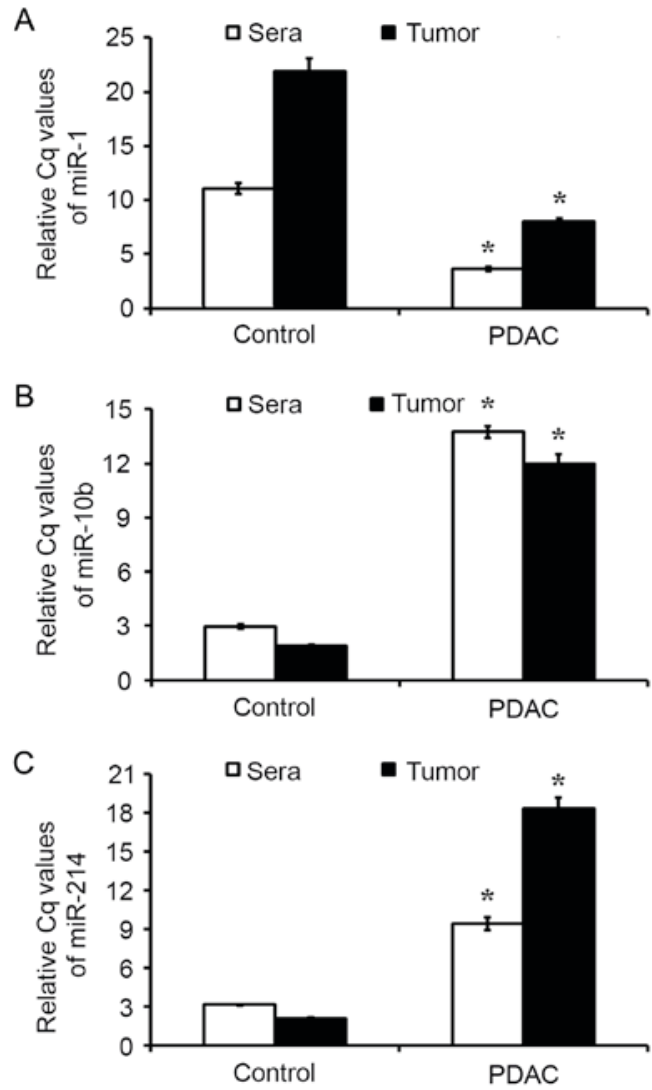

Figure 3. miRNA expression as detected using RT-qPCR. Expression levels of (A) miR-1, (B) miR-10b and (C) miR-214 in sera and tumor samples from patients with PDAC and controls were estimated by RT-qPCR. Results are presented as the mean \pm standard error of the mean. ${ }^{*} \mathrm{P}<0.05$ vs. control. RT-qPCR, reverse transcription-quantitative polymerase chain reaction; miR, microRNA; PDAC, pancreatic ductal adenocarcinoma.

miR-1 (Fig. 4A and D), miR-10b (Fig. 4B and E) and miR-214 (Fig. 4C and F) in the cytoplasm of PDAC tumor cells. 
miR-1
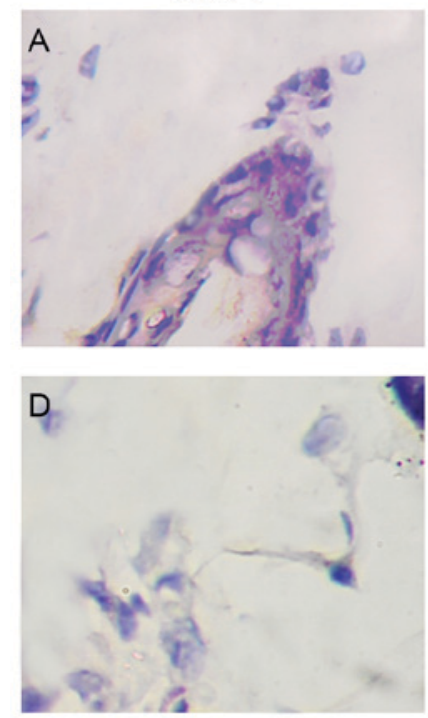

miR-10b
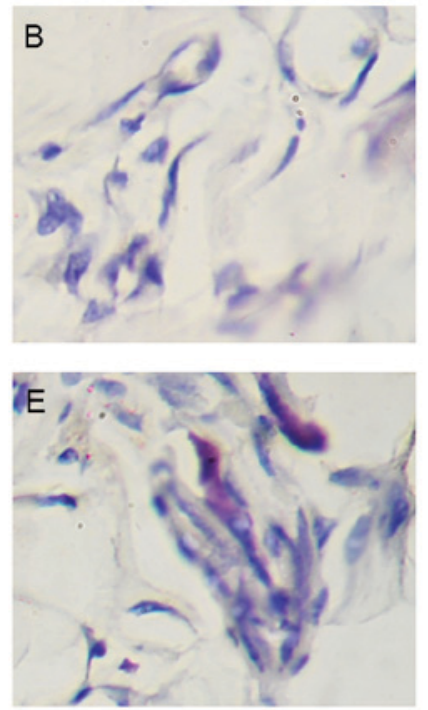
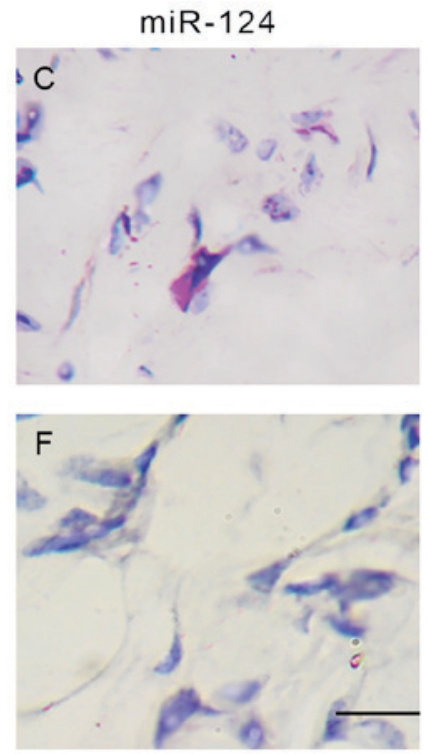

Figure 4. ISH positive staining of miR-1, miR-10b and miR-214 in PDAC and normal adjacent tissues. (A) ISH staining of miR-1 in normal tissue. (B) ISH staining of miR-10b innormal tissue. (C) ISH staining of miR-214 in normal tissue. (D) ISH staining of miR-1 in PDAC tissue. (E) ISH staining of miR-10b in PDAC tissue. (F) ISH staining of miR-214 in PDAC tissue. ISH signaling for miR-1 was observed to be weak in PDAC tissues, but marked in normal adjacent tissues. There was marked positive staining for miR-10b and miR-214 in PDAC tissues compared with that in normal adjacent tissues. Magnification, $\mathrm{x} 400$. ISH, in situ hybridization; miR, microRNA; PDAC, pancreatic ductal adenocarcinoma.

Spearman's analysis indicated that negative ISH miR-1 status was correlated with the tumor size $(r=0.661, \mathrm{P}<0.01)$, lymph node metastasis $(\mathrm{r}=0.399, \mathrm{P}<0.05)$ and the pathological tumor node metastasis ( $\mathrm{pTNM}$ ) stages $(24)(\mathrm{r}=0.327, \mathrm{P}<0.05)$ in these 43 cancer samples (Table I). Within a period of 60 months (5 years) follow-up, 10 PDAC-associated mortalities occurred, all of which were of patients with miR-1-negative tumors. The 60-month survival rate of pTNM stage 1 was $>92 \%$, whereas it was $<10 \%$ in patients with pTNM stage III-IV. Kaplan-Meier estimator analysis of the overall survival rate based on tissue miR-1 expression in the patients with a follow-up period of 60 months (Fig. 5). In the entire cohort, the overall survival rate of patients with miR-1-positive tumors was significantly increased compared with that of those with miR-1-negative tumors (82.53 vs. $23.25 \%$; log-rank test: $\chi^{2}=21.63, \mathrm{P}=0.00005$; Fig. 5A).

The results also demonstrated that the positive ISH staining of miR-214 was correlated with the tumor size $(\mathrm{r}=0.601, \mathrm{P}<0.01)$, histological grade $(\mathrm{r}=0.407, \mathrm{P}<0.01)$, lymph node metastasis $(\mathrm{r}=0.532, \mathrm{P}<0.01)$ and $\mathrm{pTNM}$ stages $(\mathrm{r}=0.327$, $\mathrm{P}<0.05)$ in the 43 tumor cases. Additionally, the overall survival rate of patients with miR-214-negative tumors was increased compared with that of those with miR-214-positive tumors (78.99 vs. $34.86 \%$; log-rank test: $\chi^{2}=11.09, \mathrm{P}=0.00061$; Table II). miR-10b staining was not observed to be correlated with the lymph node metastasis, the pTNM stages or the 5-year survival rate in the present study (data not shown).

\section{Discussion}

The results of the present study revealed expressional differential in miRNA profiles between healthy controls and patients with PDAC, either in sera or in tumor samples. In sera from the groups of patients with PDAC, expression levels of 27 miRNAs were different compared with healthy controls, in
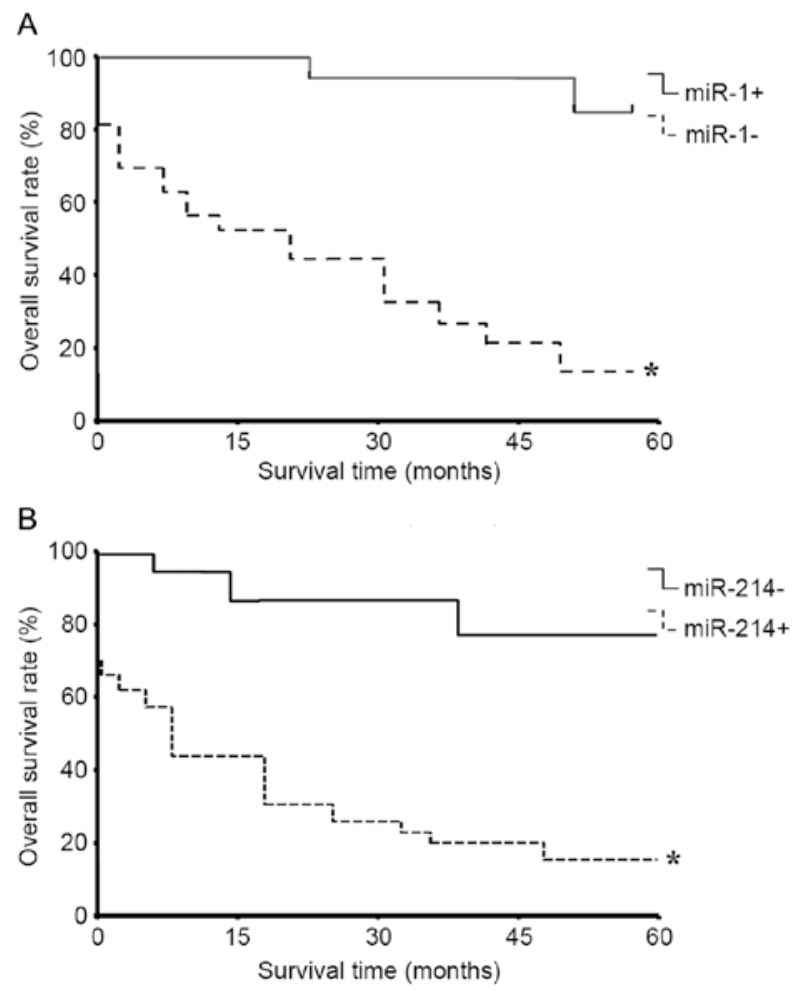

Figure 5. Survival curves (5-year) of patients with PDAC with miR-1 and miR-214 ISH status. (A) Patients with PDAC who were negative for miR-1, as determined by ISH, demonstrated significantly poorer 5 -year survival compared with those with positive ISH results ( ${ }^{\mathrm{P}}<0.05$ vs. miR-1 positive samples). (B) Patients with miR-214-positive ISH signaling exhibited significantly poorer survival compared with those with negative ISH signaling ( ${ }^{*} \mathrm{P}<0.05$ vs. miR-214 negative samples). ISH, in situ hybridization; miR, microRNA; PDAC, pancreatic ductal adenocarcinoma.

which 14 miRNAs were upregulated and another 13 miRNAs were downregulated. In tumor tissue obtained from patients 
Table I. Correlation between miR-1 status detected by ISH in pancreatic ductal adenocarcinoma tissue and clinicopathological features.

\begin{tabular}{|c|c|c|c|c|}
\hline \multirow{2}{*}{$\begin{array}{l}\text { Clinicopathological } \\
\text { feature }\end{array}$} & \multicolumn{4}{|c|}{ miR-1 ISH staining } \\
\hline & Negative $(n=32)$ & Positive $(\mathrm{n}=11)$ & $\mathrm{r}_{\mathrm{s}}$ & P-value \\
\hline Sex & & & 0.127 & 0.740000 \\
\hline Male & 17 & 5 & & \\
\hline Female & 15 & 6 & & \\
\hline Tumor size, $\mathrm{cm}$ & $3.348 \pm 0.44$ & $1.251 \pm 0.34$ & 0.661 & 0.000013 \\
\hline Histological grade & & & 0.114 & 0.120000 \\
\hline G1 & 13 & 2 & & \\
\hline $\mathrm{G} 2$ & 10 & 5 & & \\
\hline G3 & 9 & 4 & & \\
\hline LN metastasis & & & 0.399 & 0.000028 \\
\hline NO & 24 & 10 & & \\
\hline $\mathrm{N} 1$ & 8 & 1 & & \\
\hline pTNM stage & & & 0.327 & 0.000542 \\
\hline I-II & 7 & 8 & & \\
\hline III & 11 & 3 & & \\
\hline IV & 14 & 0 & & \\
\hline
\end{tabular}

Tumor size data are expressed as the mean. P-values were calculated by Spearman's rank correlation test (n=43). ISH, in situ hybridization; miR, microRNA; TNM, tumor node metastasis; LN, lymph node.

Table II. Correlation between miR-214 status detected by ISH in pancreatic ductal adenocarcinoma tissues and clinicopathological features.

\begin{tabular}{|c|c|c|c|c|}
\hline \multirow{2}{*}{$\begin{array}{l}\text { Clinicopathological } \\
\text { feature }\end{array}$} & \multicolumn{4}{|c|}{ miR-214 ISH staining } \\
\hline & Negative $(n=9)$ & Positive $(n)=34$ & $\mathrm{r}_{\mathrm{s}}$ & P-value \\
\hline Sex & & & 0.102 & 0.4900000 \\
\hline Male & 4 & 15 & & \\
\hline Female & 5 & 19 & & \\
\hline Tumor size, $\mathrm{cm}$ & $1.882 \pm 0.24$ & $3.118 \pm 0.52$ & 0.601 & 0.0000008 \\
\hline Histological grade & & & 0.407 & 0.0000015 \\
\hline G1 & 5 & 4 & & \\
\hline G2 & 3 & 17 & & \\
\hline G3 & 1 & 13 & & \\
\hline LN metastasis & & & 0.532 & 0.0000001 \\
\hline N0 & 7 & 6 & & \\
\hline N1 & 2 & 28 & & \\
\hline pTNM stage & & & 0.327 & 0.000042 \\
\hline I-II & 7 & 5 & & \\
\hline III & 2 & 16 & & \\
\hline IV & 0 & 13 & & \\
\hline
\end{tabular}

Tumor size data are expressed as mean. P-values were calculated by Spearman's rank correlation test (n=43). ISH, in situ hybridization; miR, microRNA; TNM, tumor node metastasis; LN, lymph node.

with PDAC, the expression levels of 23 miRNAs were different from the adjacent normal tissues, in which 12 miRNAs were upregulated and the remaining 11 miRNAs were downregulated. In particular, the results of microarray analysis revealed 
that miR-1, miR-10b and miR-214 expression was significantly different in the PDAC group compared with either in the sera samples or in tumor tissues. Data from RT-qPCR confirmed the upregulation of miR-10b and miR-214, and the down-regulation of miR-1, either in the sera or in the tumor samples. These results revealed that the dysregulated miR-1 and miR-214 in PDAC tissues were associated with the clinicopathological features and survival rate of patients with PDAC.

Evidence suggests that miRNA expression appears to be cell type- and disease-specific, and may be used for the classification of certain cancer histotypes, unlike most currently available biomarkers (25). Subsequent studies have demonstrated that various miRNAs were aberrantly expressed in PCC, and that these aberrant expression patterns accurately differentiated PCC from benign pancreatic tissues $(26,27)$. The results of the present study revealed that, compared with normal controls, several miRNAs were dysregulated in sera and tumor tissues from patients with PDAC, which indicated their potential roles in the pathogenic progress of PDAC.

The results of the present study also confirmed that the downregulation of miR-1, in sera and tumor tissues samples from patients with PDAC, was associated with the clinicopathological features exhibited by these patients. The carcinogenic role of miR-1 has been demonstrated in various types of cancer. Zhu and Wang (28) suggested that miR-1 was frequently decreased in clinical osteosarcoma (OS) tumor tissues and involved in the anticancer effect induced by (-)-epigallocatechin-3-gallate (28). An additional study demonstrated that miR-1 served a negative regulatory role in the proliferation of colon cancer by targeting baculoviral inhibitor of apoptosis protein repeat-containing protein 1 (29). Previous data also revealed that downregulated miR-1 was: i) Associated with colon cancer pathways and correlated with cyto- or chemokine expression (30); ii) correlated with involvement of lymph nodes, histological classification and vessel invasion of esophageal squamous cell carcinoma by binding its targeted gene LIM and Src homology 3 protein 1 and transgelin 2 (31); and iii) associated with an aggressive phenotype of human breast carcinoma (17). Downregulated hsa-miR-1 was indicated to be inversely associated with aggressiveness in breast cancer $(32,33)$. A functional study revealed that enforced overexpression of miR-1 in renal cancer cells inhibited proliferation and metastasis in vitro and in vivo (34). A previous study also demonstrated that miR-1 inhibitors significantly reduced PCC endothelial cells migration, but not proliferation (35). The results of the present study demonstrated the tumor suppressor role of miR-1 in the pathogenic progress of PDAC.

Among the two upregulated miRNAs in the sera and tumor samples, miR-214 has been previously identified to be associated with mouse pancreas development (36). The present study demonstrated that miR-214 was upregulated in patients with PDAC, and was correlated with the tumor size, histological grade, pTNM stage, lymph node metastasis and the overall survival rate (5-year) of patients with PDAC. It has been suggested previously that miR-214 was involved in the murine aging process (37), that it modulated Hedgehog signaling to specify muscle cell fate (38), induced cell survival and cisplatin resistance by targeting phosphatase and tensin homolog in human ovarian cancer (38) and negatively regulated HeLa cell proliferation and increased the survival and activity rates of T cells $(39,40)$. A previous study indicated that miR-214 functioned as either an oncogene or a tumor suppressor in different types of cancer (41). It has also been identified that the expression level of miR-214 was upregulated in gastric cancer tissues compared with matched normal tissues, and that the miR-214 expression level was significantly associated with clinical progression and poor prognosis (42). In addition, miR-214 expression was increased in PCC tissues compared with matched benign pancreatic tissues, and the overexpression of miR-214 decreased the sensitivity of the PCC cells to gemcitabine (GEM) by targeting inhibitor of growth family member 4 mRNA (43). It was also suggested that miR-214 promoted the survival of PCC cells and GEM resistance, which may be associated with the poor response to chemotherapy observed in patients with PCC (41). In combination with the results from the present study, previous data concerning the tumor suppressive role of miR-214 were confirmed. The results of the present study suggested that dysregulated miR-214, as a negative regulator, was associated with the clinicopathological features and prognosis indices of patients with PDAC. Furthermore, increased expression of miR-10b has been demonstrated in glioma and metastatic PCC $(44,45)$. Notably, increased miR-10b blood sera levels have been described in gastric cancer, non-small lung cancer and metastatic breast cancer $(46,47)$, which was also observed in the present study and suggested its oncogenic role in PDAC.

In conclusion, the results of the microarray analysis revealed that miR-1, miR-10b and miR-214 were significantly altered in the PDAC sera or tumor tissue samples. Data from RT-qPCR confirmed the dysregulation of miR-1, miR-10b and miR-214 in sera and tumor samples. Analysis revealed that the dysregulated miR-1 and miR-214 in PDAC tissues were associated with the clinicopathological features and 5-year survival rates of patients with PDAC. The results of the present study identified useful markers, miR-1 and miR-214, for determining the prognosis of patients with PDAC by noninvasive methods.

\section{References}

1. Verbeke C, Löhr M, Karlsson JS and Del Chiaro M: Pathology reporting of pancreatic cancer following neoadjuvant therapy: Challenges and uncertainties. Cancer Treat Rev 41: 17-26, 2015.

2. Rahib L, Smith BD, Aizenberg R, Rosenzweig AB, Fleshman JM and Matrisian LM: Projecting cancer incidence and deaths to 2030: The unexpected burden of thyroid, liver and pancreas cancers in the United States. Cancer Res 74: 2913-2921, 2014.

3. Hidalgo M, Cascinu S, Kleeff J, Labianca R, Löhr JM, Neoptolemos J, Real FX, Van Laethem JL and Heinemann V: Addressing the challenges of pancreatic cancer: Future directions for improving outcomes. Pancreatology 15: 8-1829, 2015.

4. Bardeesy N and DePinho RA: Pancreatic cancer biology and genetics. Nat Rev Cancer 2: 897-909, 2002.

5. Partensky C: Toward a better understanding of pancreatic ductal adenocarcinoma: Glimmers of hope? Pancreas 42: 729-739, 2013.

6. Herreros-Villanueva M, Zhang JS, Koenig A, Abel EV, Smyrk TC Bamlet WR, de Narvajas AA, Gomez TS, Simeone DM, Bujanda L and Billadeau DD: SOX2 promotes dedifferentiation and imparts stem cell-like features to pancreatic cancer cells. Oncogenesis 2: e61, 2013.

7. Han JB, Sang F, Chang JJ, Hua YQ, Shi WD, Tang LH and Liu LM: Arsenic trioxide inhibits viability of pancreatic cancer stem cells in culture and in a xenograft model via binding to SHHGli. Onco Targets Ther 6: 1129-1138, 2013. 
8. Wang X, Liu Q, Hou B, Zhang W, Yan M, Jia H, Li H, Yan D, Zheng F, Ding W, et al: Concomitant targeting of multiple key transcription factors effectively disrupts cancer stem cells enriched in side population of human pancreatic cancer cells. PLoS One 8: e73942, 2013.

9. Lu Y, Zhu H, Shan H, Lu J, Chang X, Li X, Lu J, Fan X, Zhu S, Wang Y, et al: Knockdown of Oct4 and Nanog expression inhibits the stemness of pancreatic cancer cells. Cancer Lett 340 113-123, 2013.

10. Hu T, Chang YF, Xiao Z, Mao R, Tong J, Chen B, Liu GC, Hong Y, Chen HL, Kong SY, et al: miR-1 inhibits progression of high-risk papillomavirus-associated human cervical cancer by targeting G6PD. Oncotarget 7: 86103-86116, 2016.

11. Hiyoshi Y, Kamohara H, Karashima R, Sato N, Imamura Y, Nagai Y, Yoshida N, Toyama E, Hayashi N, Watanabe M, et al: MicroRNA-21 regulates the proliferation and invasion in esophageal squamous cell carcinoma. Clin Cancer Res 15: 1915-1922, 2009.

12. Moriyama T, Ohuchida K, Mizumoto K, Yu J, Sato N, Nabae T, Takahata S, Toma H, Nagai E and Tanaka M: MicroRNA-21 modulates biological functions of pancreatic cancer cells including their proliferation, invasion and chemoresistance. Mol Cancer Ther 8: 1067-1074, 2009.

13. Park EY, Chang E, Lee EJ, Lee HW, Kang HG, Chun KH Woo YM, Kong HK, Ko JY, Suzuki H, et al: Targeting of miR34a-NOTCH1 axis reduced breast cancer stemness and chemoresistance. Cancer Res 74: 7573-7582, 2014.

14. Volinia S, Nuovo G, Drusco A, Costinean S, Abujarour R, Desponts C, Garofalo M, Baffa R, Aeqilan R, Maharry K, et al: Pluripotent stem cell miRNAs and metastasis in invasive breast cancer. J Natl Cancer Inst 106: 1-8, 2014.

15. Le Large TY, Meijer LL, Mato Prado M, Kazemier G, Frampton AE and Giovannetti E: Circulating microRNAs as diagnostic biomarkers for pancreatic cancer. Expert Rev Mol Diagn 15: 1-5, 2015

16. Hou FQ, Lei XF, Yao JL, Wang YJ and Zhang W: Tetraspanin 1 is involved in survival, proliferation and carcinogenesis of pancreatic cancer. Oncol Rep 34: 3068-3076, 2015.

17. Gustafsson OJ, Briggs MT, Condina MR, Winderbaum LJ, Pelzing M, McColl SR, Everest-Dass AV, Packer NH and Hoffmann P: MALDI imaging mass spectrometry of N-linked glycans on formalin-fixed paraffin-embedded murine kidney. Anal Bioanal Chem 407: 2127-2139, 2015.

18. Brunner TB, Merkel S, Grabenbauer GG, Meyer T, Baum U, Papadopoulos T, Sauer R and Hohenberger W: Definition of elective lymphatic target volume in ductal carcinoma of the pancreatic head based on histopathologic analysis. Int J Radiat Oncol Biol Phys 62: 1021-1029, 2015.

19. Green MR and Sambrook J: Molecular Cloning: A Laboratory Manual. 4th Edition. Cold Spring Harbor Laboratory Press, pp139-151, 2013.

20. Livak KJ and Schmittgen TD: Analysis of relative gene expression data using real-time quantitative PCR and the 2(-Delta Delta C(T)) Method. Methods 25: p402-p408, 2001.

21. Ouchi Y, Banno Y, Shimizu Y, Ando S, Hasegawa H, Adachi K and Iwamoto T: Reduced adult hippocampal neurogenesis and working memory deficits in the Dgcr8-deficientmouse model of 22q11.2 deletion-associated schizophrenia can be rescued by IGF2. J Neurosci 33: 9408-9019, 2013.

22. Minemura H, Takagi K, Miki Y, Shibahara Y, Nakagawa S, Ebata A, Watanabe M, Ishida T, Sasano H and Suzuki T: Abnorma expression of miR-1 in breast carcinoma as a potent prognostic factor. Cancer Sci 106: 1642-1650, 2015.

23. Mansour A, Chatila R, Bejjani N, Dagher C and Faour WH: A novel xylene-free deparaffinization method for the extraction of proteins from human derived formalin-fixed paraffin embedded (FFPE) archival tissue blocks. MethodsX 1: 90-95, 2014.

24. Chen XP and Wang JP: Surgery. 8th Edition. People's Medical Press, pp302-312, 2013.

25. Rosenfeld N, Aharonov R, Meiri E, Rosenwald S, Spector Y, Zepeniuk M, Benjamin H, Shabes N, Tabak S, Levy A, et al: MicroRNAs accurately identify cancer tissue origin. Nat Biotechnol 26: 462-469, 2008.

26. Habbe N, Koorstra JB, Mendell JT, Offerhaus GJ, Ryu JK, Feldmann G, Mullendore ME, Goggins MG, Hong SM and Maitra A: MicroRNA miR-155 is a biomarker of early pancreatic neoplasia. Cancer Biol Ther 8: 340-346, 2009.

27. Bloomston M, Frankel WL, Petrocca F, Volinia S, Alder H, Hagan JP, Liu CG, Bhatt D, Taccioli C and Croce CM: MicroRNA expression patterns to differentiate pancreatic adenocarcinoma from normal pancreas and chronic pancreatitis. JAMA 297: 1901-1908, 2007
28. Zhu K and Wang W: Green tea polyphenol EGCG suppresses osteosarcoma cell growth through upregulating miR-1. Tumour Biol 37: 4373-4382, 2015.

29. Xu X, Wu X, Jiang Q, Sun Y, Liu H, Chen R and Wu S: Downregulation of microRNA-1 and microRNA-145 contributes synergistically to the development of colon cancer. Int J Mol Med 36: 1630-1638, 2015.

30. Pathak S, Meng WJ, Nandy SK, Ping J, Bisgin A, Helmfors L, Waldmann P and Sun XF: Radiation and SN38 treatments modulate the expression of microRNAs, cytokines and chemokines in colon cancer cells in a p53-directed manner. Oncotarget 6: 44758-44780, 2015.

31. Du YY, Zhao LM, Chen L, Sang MX, Li J, Ma M and Liu JF: The tumor-suppressive function of miR-1 by targeting LASP1 and TAGLN2 in esophageal squamous cell carcinoma. J Gastroenterol Hepatol 31: 384-393, 2015.

32. Liu T, Hu K, Zhao Z, Chen G, Ou X, Zhang H, Zhang X, Wei X, Wang D, Cui M et al: MicroRNA-1 down-regulates proliferation and migration of breast cancer stem cells by inhibiting the Wnt/ß-catenin pathway. Oncotarget 6: 41638-41649 2015.

33. Liu R, Li J, Lai Y, Liao Y, Liu R and Qiu W: Hsa-miR-1 suppresses breast cancer development by down-regulating K-ras and long non-coding RNA MALAT1. Int J Biol Macromol 81: 491-497, 2015.

34. Xiao H, Zeng J, Li H, Chen K, Yu G, Hu J, Tang K, Zhou H, Huang Q, Li A, et al: MiR-1 downregulation correlates with poor survival in clear cell renal cell carcinoma where it interferes with cell cycle regulation and metastasis. Oncotarget 6: 13201-13215, 2015.

35. Li L, Li B, Chen D, Liu L, Huang C, Lu Z, Lun L and Wan X: miR-139 and miR-200c regulate pancreatic cancer endothelial cell migration and angiogenesis. Oncol Rep 34: 51-58, 2015.

36. Lynn FC, Skewes-Cox P, Kosaka Y, McManus MT, Harfe BD and German MS: MicroRNA expression is required for pancreatic islet cell genesis in the mouse. Diabetes 56: 2938-2945, 2007.

37. Esquela-Kerscher A and Slack FJ: Oncomirs-microRNAs with a role in cancer. Nat Rev Cancer 6: 259-269, 2006.

38. Meng F, Henson R, Wehbe-Janek H, Ghoshal K, Jacob ST and Patel T: MicroRNA-21 regulates expression of the PTEN tumor suppressor gene in human hepatocellular cancer. Gastroenterology 133: 647-658, 2007.

39. Yang Z, Chen S, Luan X, Li Y, Liu M, Li X, Liu T and Tang H: MicroRNA-214 is aberrantly expressed in cervical cancers and inhibits the growth of HeLa cells. IUBMB Life 61: 1075-1082, 2009.

40. Jindra PT, Bagley J, Godwin JG and Iacomini J: Costimulationdependent expression of microRNA-214 increases the ability of T cells to proliferate by targeting Pten. J Immunol 185: 990-997, 2010.

41. Zhang XJ, Ye H, Zeng CW, He B, Zhang $\mathrm{H}$ and Chen YQ: Dysregulation of miR-15a and miR-214 in human pancreatic cancer. J Hematol Oncol 3: 46, 2010.

42. Yang TS, Yang XH, Wang XD, Wang YL, Zhou B and Song ZS: MiR-214 regulate gastric cancer cell proliferation, migration and invasion by targeting PTEN. Cancer Cell Int 13: 68, 2013.

43. Flynt AS, Li N, Thatcher EJ, Solnica-Krezel L and Patton JG: Zebrafish miR-214 modulates Hedgehog signaling to specify muscle cell fate. Nat Genet 39: 259-263, 2007.

44. Sasayama T, Nishihara M, Kondoh T, Hosoda K and Kohmura E: MicroRNA-10b is overexpressed in malignant glioma and associated with tumor invasive factors, uPAR and RhoC. Int J Cancer 125: 1407-1413, 2009.

45. Nakata K, Ohuchida K, Mizumoto K, Kayashima T, Ikenaga N, SakaiH,Lin C,FujitaH,OtsukaT, Aishima S, et al: MicroRNA-10b is overexpressed in pancreatic cancer, promotes its invasiveness and correlates with a poor prognosis. Surgery 150: 916-922, 2011.

46. Roth C, Kasimir-Bauer S, Pantel K and Schwarzenbach H: Screening for circulating nucleic acids and caspase activity in the peripheral blood as potential diagnostic tools in lung cancer. Mol Oncol 5: 281-291, 2011

47. Nishida N, Yamashita S, Mimori K, Sudo T, Tanaka F, Shibata K, Yamamoto H, Ishii H, Doki Y and Mori M: MicroRNA-10b is a prognostic indicator in colorectal cancer and confers resistance to the chemotherapeutic agent 5-fluorouracil in colorectal cancer cells. Ann Surg Oncol 19: 3065-3071, 2012. 